Interfaces and Free Boundaries 14 (2012), 145-152

DOI 10.4171/IFB/276

\title{
Regular solutions to a monodimensional model with discontinuous elliptic operator
}

\author{
Piotr BogusŁaw Mucha \\ Institute of Applied Mathematics and Mechanics, Warsaw University, ul. Banacha 2, 02-097 \\ Warszawa, Poland \\ E-mail:p.mucha@mimuw.edu.pl
}

[Received 14 December 2010 and in revised form 13 February 2012]

\begin{abstract}
The note examines qualitative behavior of solutions to a monodimensional nonlinear elliptic equation $\frac{d}{d x}\left(u_{x}+\operatorname{sgn} u_{x}\right)=f$ with Dirichlet boundary data. This simple example explains the phenomenon of facets - flat regions of solutions, characteristic for models arising from theories of crystal growth and image processing.
\end{abstract}

2010 Mathematics Subject Classification: Primary 35J75, 35J15; Secondary 74E15, 94A08.

Keywords: Singular elliptic operator, discontinuity, facets, qualitative analysis, structure of solutions.

\section{Introduction}

The subject of this note is qualitative analysis of a monodimensional version of a class of nonlinear elliptic systems

$$
\operatorname{div} F(\nabla u)=f \text { in } \Omega, \quad u=g \text { at } \partial \Omega,
$$

where $F$ is a monotone function. This classical nonlinear problem is well studied in the literature $[2,13]$, but in the case of discontinuous function $F$ the theory delivers only very general result, we mention here $[4,7,15,18]$, which of course do not represent the whole theory. From the viewpoint analysis of one-dimensional case seems to be very promising [1, 6]. It shall give hints how to deal with multi-dimensional case and what type of properties one can expect. Realistic models arising from applications are usually so complex that it is almost impossible to point a key analytical obstacle.

On the other hand monodimensional version of (1) can be found in models arising from the theories of crystal growth and image processing $[3,5,8,14,16]$, where description of interfaces, like free boundaries or edges, is the heart of studied phenomena. In modeling, qualitative analysis of solutions is irreplaceable for effective application to a particular model. In many of such systems, the discontinuity of $F$ causes appearance of facets - flat parts of solutions. They are the kernel of analysis of behavior of solutions to the total variation equation or the weighted mean curvature flows $[9,12,17,19]$. To try to understand this phenomenon we would like to study a simple model. Even for the simplification, studied in this note, we obtain some unexpected properties. In particular we make clear the appearance condition of facets in a class of typical solutions. Analysis of such models seems to be the best way to understand deeper the nature of this unusual fascinating phenomenon. From the point of view of the theory of crystal growth, our equation is related to a free boundary problem for a model with anisotropy which is strictly convex but not smooth, so called "lens" crystal for which the structural unit is lens-shaped. Mathematical theory of such problems is an almost untouched field. 


\section{The results}

We aim at solving the following monodimensional elliptic problem

$$
\frac{d}{d x} L\left(u_{x}\right)=f \text { in }[0,1], \quad u(0)=A, \quad u(1)=B .
$$

Within this note we assume that $L(\cdot)$ is in the following form

$$
L(w)=w+\operatorname{sgn} w=\left\{\begin{array}{lll}
w+1 & \text { for } & w>0 \\
{[-1,1]} & \text { for } & w=0 \\
w-1 & \text { for } & w<0
\end{array}\right.
$$

We treat $L(\cdot)$ as a maximal multivalued function, i.e., for each $x \in \mathbb{R}$ we have $L(x)=$ $\left[\lim _{z \rightarrow x^{-}} L(z), \lim _{z \rightarrow x^{+}} L(z)\right]$.

Now let us state the first result proved here, concerning the issue of existence and regularity.

THEOREM 1 Let $f \in L_{\infty}(0,1)$, then there exists a unique solution to (2) such that $u \in W_{\infty}^{2}(0,1)$ and $u(0)=A, u(1)=B$ in the following weak meaning

$$
\left(\sigma, \phi_{x}\right)=-(f, \phi) \text { for each } \phi \in C_{0}^{\infty}(0,1)
$$

and $\sigma \in L_{1}(0,1)$ with $\left.\sigma\left(x_{*}\right) \in L\left(u_{x}\right)\right|_{x=x_{*}}$ a.e. $x_{*} \in(0,1)$, where $(\cdot, \cdot)$ is the usual inner product in $L_{2}(0,1)$, defined as $(u, v)=\int_{0}^{1} u v d x$ for $u, v \in L_{2}(0,1)$.

A closer look at the above theorem suggests that it is rather expected. However it defines us optimal regularity of solutions to (4), too. The $W_{\infty}^{2}$ class is relatively high, but it seems to be the best possible - provided that even $f$ is smooth. A key information from Theorem 1 is that the derivative of solutions is continuous, even Lipschitz continuous. Of course, a question to ask whether such approach to the issue of regularity to solutions to (4) is suitable, reminds open. Another question is a meaning of solutions. The second derivative is well defined, even bounded, but all known definitions at the set $\left\{u_{x}=0\right\}$ fail. Even the approach via "almost classical" solutions developed by [17] seems to be not appropriate.

The next result describes precisely shape of solutions, particularly it determines appearance of facets. In order to obtain the full characterization of this phenomenon we restrict ourself to the case of nonnegative data $f$. Additionally we fix boundary data $(u(0)=0$ and $u(1)=1)$ to make the obtained characterization more readable.

THEOREM 2 Let $A=0$ and $B=1, f \in L_{\infty}(0,1)$ and $f \geqslant 0$, then $u$ - the weak solution to (2) is convex. Furthermore the shape of solution is described by one of three possibilities below:

- $u$ is strictly monotone, provided

$$
\int_{0}^{1} d \tau \int_{0}^{\tau} f(s) d s<1
$$

- $u$ is monotone with a flat part (a facet) touching point $x=0$, i.e.

$$
u= \begin{cases}0 & \text { for } x \in(0, b] \\ \text { strictly increasing } & \text { for } x \in(b, 1),\end{cases}
$$


for some $b \in[0,1)$, provided existence of $d \in[0,1)$ such that

$$
\int_{d}^{1} d \tau \int_{d}^{\tau} f(s) d s=1 \text { and } \int_{0}^{d} f(s) d s \leqslant 2 ;
$$

- $u$ has an interior minimum realized on a nondegenerate interval $[a, b] \subset(0,1)$, i.e.,

$$
u= \begin{cases}\text { strictly decreasing } & \text { for } \quad x \in(0, a), \\ \text { constant } & \text { for } x \in[a, b], \\ \text { strictly increasing } & \text { for } x \in(b, 1),\end{cases}
$$

provided existence of $d \in(0,1)$ such that

$$
\int_{d}^{1} d \tau \int_{d}^{\tau} f(s) d s=1 \text { and } \int_{0}^{d} f(s) d s>2 .
$$

The above result describes structure of solutions to (2). It provides necessary and sufficient conditions of generation of interior facets. In case of general $f$, the structure of solutions is of course more complex, however local behavior of solutions with fixed convexity is the same as described by Theorem 2 . As an addition we obtain a restriction of possible regularity of solutions. For $f \equiv$ constant, we obtain $u_{x x}$ just in $L_{\infty}$, provided that this constant is sufficiently large, in order to fulfill condition (7). Thus, the regularity given by Theorem 1 is optimal.

The third result points an interesting phenomenon which holds for the case $u(0)=u(1)$. It is a limit version of Theorem 2, but more spectacular.

Theorem 3 Let $A=B=0, f \in L_{\infty}(0,1)$ and $f \geqslant 0$, then solution to (2) is:

- $u \equiv 0$, provided $\int_{0}^{1} f(s) d s \leqslant 2$;

- $u$ has an interior minimum realized on a nondegenerate interval $[a, b] \subset(0,1)$, i.e.,

$$
u= \begin{cases}\text { strictly decreasing } & \text { for } x \in(0, a), \\ \text { constant } & \text { for } x \in[a, b], \\ \text { strictly increasing } & \text { for } x \in(b, 1),\end{cases}
$$

$$
\text { provided } \int_{0}^{1} f(s) d s>2 .
$$

The first part of Theorem 3 may be generalized of the case of $f$ with variable sign.

Theorem 4 Let $A=B=0, f \in L_{\infty}(0,1)$, then weak solution to (2) is trivial, i.e. $u \equiv 0$, provided $0 \leqslant \int_{0}^{x} f(s) d s \leqslant 2$ for all $x \in[0,1]$.

The above results show that for sufficiently small data $f$, or rather suitable, solutions to (2) are trivial. It is a consequence of jumps of function $L(\cdot)$. The singularity is so strong that it absorbs the whole influence coming from $f$. Similar effects have been observed in analysis of a model of crystal growth. Equation (1) has a variational structure which is similar to that of determining the crystalline curvature of a facet in case there is a driving due to the influx of diffusing matter, see Formula (1.8) in [10]. The authors showed there results which can be stated as follows, if the driving, 
i.e., influx is small, (cf. [10, Corollary 4.13]), then the facets is stable. The original formulation is different: if the facet is smaller than a critical length, then it is stable, see [10, Theorem 4.8, Theorem 4.14], [11, Theorem 4.7, Corollary 4.9].

In what follows we are trying to use standard notation. Below we prove subsequently our main results.

Proof of Theorem 1. In order to prove the existence we follow a classical approach via a regularization of the function $L$. We show few details to the proof since it will be useful in the proof of the next theorem being the main contribution of this note.

Given $\epsilon>0$, let us define

$$
L_{\epsilon}(w)=w+\operatorname{sgn}_{\epsilon}(w)=w+\delta_{\epsilon} * \operatorname{sgn} w .
$$

Functions $\delta_{\epsilon}$ stand for a smooth approximation of the Dirac measure $\left(\delta_{\epsilon} \rightarrow \delta_{0}\right.$ in $\left.D^{\prime}(\mathbb{R})\right)$. Definition (8) follows that $L_{\epsilon}$ is strictly increasing and $L_{\epsilon}^{\prime}=1+\delta_{\epsilon} \leqslant \frac{C}{\epsilon}$. In addition for $|w|>\epsilon$ we keep $L_{\epsilon}(w)=L(w)$.

Examine the approximative system of (2)

$$
\frac{d}{d x} L_{\epsilon}\left(u_{x}^{\epsilon}\right)=f \quad \text { in }[0,1], \quad u^{\epsilon}(0)=A, \quad u^{\epsilon}(1)=B .
$$

A proof of existence to the above problem follows immediately from the Schauder fixed point theorem. Define a map $T: C^{1+\alpha}(0,1) \rightarrow C^{1+\alpha}(0,1)$ for some fixed $\alpha \in(0,1)$ such that $T(\tilde{u})=$ $u$, where $u$ is a solution to

$$
\left(1+\delta_{\epsilon}\left(\tilde{u}_{x}\right)\right) u_{x x}=f \quad \text { in }[0,1], \quad u(0)=A, \quad u(1)=B .
$$

Since $1+\delta_{\epsilon}\left(\tilde{u}_{x}\right) \geqslant 1$, we find that $\left|u_{x x}\right| \leqslant|f|$ pointwisely a.e. thus we get $\|u\|_{W_{\infty}^{2}(0,1)} \leqslant$ $C\left(\|f\|_{L_{\infty}(0,1)}+1\right)$, where the constant $C$ depends on the boundary data $A$ and $B$. Solvability of (10) is immediate. It is clear that for sufficiently large ball $B$ in $C^{1+\alpha}$, we have $T B \subset B$ and the estimate in the $W_{\infty}^{2}$-norm yields the necessary compactness. Thus we proved existence to system (9) with a uniform in $\epsilon$ bound

$$
\left\|u^{\epsilon}\right\|_{W_{\infty}^{2}(0,1)} \leqslant C\left(\|f\|_{L_{\infty}(0,1)}+1\right) .
$$

The uniqueness to (9) follows from strict monotonicity of $L_{\epsilon}$.

Now we are in a good position to pass to the limit. By (11) we choose a subsequence

$$
u^{\epsilon} \rightarrow^{*} u \text { in } W_{\infty}^{2}(0,1) \text { with }\|u\|_{W_{\infty}^{2}(0,1)} \leqslant C\left(\|f\|_{L_{\infty}(0,1)}+1\right)
$$

and

$$
L_{\epsilon}\left(u_{x}^{\epsilon}\right) \rightarrow^{*} \sigma \text { in } L_{\infty}(0,1) \text { with }\|\sigma\|_{L_{\infty}(0,1)} \leqslant C\left(\|f\|_{L_{\infty}(0,1)}+1\right) .
$$

Let us check whether $(u, \sigma)$ fulfills (4). Given $\delta>0$, we consider the set

$$
Q_{\delta}=\left\{x \in(0,1):\left|u_{x}(x)\right|>\delta\right\} .
$$

Then thanks to convergence of $\left\{u_{x}^{\epsilon}\right\}$ in a strong topology $C([0,1])$, we are able to find $0<\epsilon_{0}<\delta / 2$ such that for $0<\epsilon<\epsilon_{0}$ there holds

$$
\left\{x \in(0,1):\left|u_{x}^{\epsilon}(x)\right|>\frac{\delta}{2}\right\} \supset Q_{\delta} \supset\left\{x \in(0,1):\left|u_{x}^{\epsilon}(x)\right|>2 \delta\right\} .
$$


Hence $L_{\epsilon}\left(u_{x}^{\epsilon}\right)=u_{x}^{\epsilon}+\operatorname{sgn} u_{x}^{\epsilon}$ on the set $Q_{\delta}$ for each $\epsilon \in\left(0, \epsilon_{0}\right)$.

Next, we consider the weak form of (9). For each $\phi \in C_{0}^{\infty}(0,1)$ regular solutions to (9) fulfill

$$
\left(L_{\epsilon}\left(u_{x}^{\epsilon}\right), \phi_{x}\right)=-(f, \phi) .
$$

Remembering that $\delta>0$ is fixed, we find

$$
\left.L_{\epsilon}\left(u_{x}^{\epsilon}\right)\right|_{\mathfrak{Q}_{\delta}}=u_{x}^{\epsilon}+\left.\operatorname{sgn} u_{x}^{\epsilon}\right|_{\boldsymbol{Q}_{\delta}} \rightarrow u_{x}+\left.\operatorname{sgn} u_{x}\right|_{\boldsymbol{Q}_{\delta}} \text { in } L_{\infty}\left(Q_{\delta}\right) \text { as } \epsilon \rightarrow 0 .
$$

On the other hand by (15) and (8)

$$
\left.L_{\epsilon}\left(u_{x}^{\epsilon}\right)\right|_{[0,1] \backslash Q_{\delta}} \subset[-1-2 \delta, 1+2 \delta] .
$$

Subsequently, as $\epsilon \rightarrow 0$

$$
\left.L_{\epsilon}\left(u_{x}^{\epsilon}\right)\right|_{[0,1] \backslash Q_{\delta}} \rightarrow^{*} \overline{L\left(u_{x}\right)} \text { in } L_{\infty}\left([0,1] \backslash Q_{\delta}\right) \text { and }\left.\overline{L\left(u_{x}\right)}\right|_{[0,1] \backslash \Theta_{\delta}} \in[-1-2 \delta, 1+2 \delta] .
$$

Here we follow the notation that $\overline{L\left(u_{x}\right)}$ denotes a weak limit of subsequence $L_{\epsilon}\left(u_{x}^{\epsilon}\right)$.

Since $\delta>0$ is chosen in an arbitrary way, we pass with $\epsilon \rightarrow 0$ in (16) and from (13), (17), (18) and (19) we conclude that the sought limit

$$
\sigma=\overline{L\left(u_{x}\right)}=\left\{\begin{array}{llr}
u_{x}+\operatorname{sgn} u_{x} & \text { for } & u_{x} \neq 0 \\
\in[-1,1] & \text { for } & u_{x}=0
\end{array}\right.
$$

fulfills the weak formulation (4), namely, $u$ given by (12) is a weak solution to (2).

The strict monotonicity of $L(\cdot)$ yields the uniqueness, since high regularity of solutions allows us to use a difference of two solutions as a test function in the weak formulation (4). Theorem 1 is proved.

Proof of Theorem 2. By considerations in the previous proof, by (10), we find that $0 \leqslant u_{x x}(x) \leqslant$ $f(x)$, so $u$ stays convex at $[0,1]$. In order to analyze three possibilities pointed by the thesis of Theorem 2 we examine the possible minimum of $u$ over $[0,1]$.

Let us assume that $u$ has an essential minimum, i.e., $\min _{x \in[0,1]} u(x)=m$ and $m<0$. Then there are two possibilities being a consequence of convexity of $u$ : either the minimum is realized at a single point or at a connected interval, say $[a, b] \subset(0,1)$. We want to exclude the first case. Assume that it holds, so there exists a point $c \in(0,1)$ such that $u(c)=m<0$ and $u$ is strictly deceasing on $(0, c)$ and strictly increasing on $(c, 1)$.

Given $\gamma>0$, define a sequence of smooth functions $\phi^{l} \in C_{0}^{\infty}(0,1)$, an approximation of characteristic function of set $(c-\gamma, c+\gamma)$, such that $\phi^{l} \rightarrow \chi_{[c-\gamma, c+\gamma]}$ a.e. pointwisely. In particular $\phi_{x}^{l} \rightarrow \delta(\cdot-(c-\gamma))-\delta(\cdot-(c+\gamma))$ in $D^{\prime}(0,1)$ as $l \rightarrow \infty$. Here $\delta(\cdot)$ denotes the Dirac measure located at point zero.

In small neighborhoods of points $c-\gamma$ and $c+\gamma$ we have $\overline{L\left(u_{x}\right)}=u_{x}$, so by (12), (17) and (20) it belongs at least to $C^{\alpha}$ for some $\alpha>0$. It is sufficient to pass to the limit with $l$ in order to use the limit value of test functions, namely the Dirac measures. So the limit applied to (4) yields

$$
\left.L\left(u_{x}\right)\right|_{x=c+\gamma}-\left.L\left(u_{x}\right)\right|_{x=c-\gamma}=\int_{c-\gamma}^{c+\gamma} f d s .
$$


By Theorem 1 we know $u_{x} \in W_{\infty}^{1}(0,1)$, so the continuity of $u_{x}$ follows that $u_{x}(c \pm \gamma) \rightarrow 0^{ \pm}$as $\gamma \rightarrow 0^{+}$, hence (21) takes the form

$$
2=\lim _{\gamma \rightarrow 0} \int_{c-\gamma}^{c+\gamma} f d s=0
$$

what can not be true as $f$ is locally integrable. To obtain (22) we shall recall that $\lim _{w \rightarrow 0^{+}} L(w)-$ $L(-w)=2$, which follows directly from definition (3).

The above analysis said that the function $u$, in the examined case, admits only interior minima realized by a nontrivial interval, we name it $[a, b]$ with $a<b$. Considering a sequence of smooth test functions $\phi^{l}$ such that $\phi^{l} \rightarrow \chi_{[a-\gamma, b+\gamma]}$, using the same arguments as for (22), we find the necessary condition

$$
2=\int_{a}^{b} f(x) d x
$$

Hence we got the first condition admitting the third case: $\int_{0}^{1} f(x) d x>2$.

Let us analyze the third case of Theorem 2 more deeply. By definition on $[a, b]$ the function is constant and the regularity guaranteed by Theorem 1 implied that $\left.u_{x}\right|_{\{a, b\}}=0$. So we find the following prescription of the solution to (4)

$$
\begin{array}{llll}
u_{x x}=f & \text { for } x \in(0, a), & u(0)=0, & u_{x}(a)=0 \\
u=m & \text { for } x \in[a, b], & & \\
u_{x x}=f & \text { for } x \in(b, 1), & u_{x}(b)=0, & u(1)=1 .
\end{array}
$$

Note that we are able to solve systems $(24)_{1,3}$, provided that $a, b$ are known. Thus

$$
\begin{array}{ll}
u(x)=-\int_{0}^{x} d \tau \int_{\tau}^{a} f(s) d s & \text { for } x \in(0, a), \\
u(x)=1-\int_{x}^{1} d \tau \int_{b}^{\tau} f(s) d s & \text { for } x \in(b, 1) .
\end{array}
$$

The last formula provides criteria which describes as three cases stated in Theorem 2:

1. Note that if (5) holds, then above analysis has no realization. Using $(25)_{2}$ we are not able to reach point $u(0)=0$, so the only solution must fulfill the following problem

$$
u_{x x}=f \quad \text { for } x \in(0,1), \quad u(0)=0, \quad u(1)=1 .
$$

In this case the only minimum is at $x=0$.

2. The second case holds if (6) is assumed. Analysis of the first part yields that in the second case there exists $\bar{d} \in[0,1)$ such that $u_{x}(\bar{d})=0$ and $u$ is strictly monotone on $[\bar{d}, 1]$. It is clear that $u(\bar{d})=\min u$ over $[0,1]$. Since considerations from the beginning of the proof excluded the case $u(\bar{d})<0$ under (6), we deduce $u(\bar{d})=0$. Recalling $u(0)=0$ we obtain the desired form of the solutions in the second case. Observer that $\bar{d}=b=d$.

3. The last point describes the case of (7). Then we have for some $m<0$

$$
-\int_{0}^{a} d \tau \int_{\tau}^{a} f(s) d s=m, \quad \int_{a}^{b} f(s) d s=2, \quad 1-\int_{b}^{1} d \tau \int_{b}^{\tau} f(s) d s=m .
$$


To find $m, a, b$ we construct the following function $\phi$ such that: for a given $b^{\prime} \in[0, d]$ we find $m^{\prime}=m^{\prime}(b)=1-\int_{b^{\prime}}^{1} d \tau \int_{b^{\prime}}^{\tau} f(s) d s$ and $b^{\prime}$ is taken to satisfy that $m^{\prime} \leqslant 0$ and $\int_{0}^{b^{\prime}} f(s) d s>0 ;$ next we find $a^{\prime} \in\left[0, b^{\prime}\right]$ by condition $\int_{a^{\prime}}^{b^{\prime}} f(s) d s=2$; and finally we put $\phi\left(b^{\prime}\right)=m^{\prime}+$ $\int_{0}^{a^{\prime}} d \tau \int_{\tau}^{a^{\prime}} f(s) d s$

Next, we observe that as we find $b_{*}$ such that $m^{\prime}\left(b_{*}\right)=0$, then $\phi\left(b_{*}\right)>0$. And $b^{*}$ such that $a^{*}=0$, then $\phi\left(b^{*}\right)<0$. Clearly the map $\phi$ is continuous, so we find $b$ such that $\phi(b)=0$, what defines the sought triplet $(m, a, b)$ from (26). Theorem 2 is proved.

Proof of Theorems 3 and 4 . The first part of Theorem 3 and Theorem 4 require just one observation. Note that putting

$$
\sigma(x)=-1+\int_{0}^{x} f(s) d s \in[-1,1], \text { then } \frac{d}{d x} \sigma=f .
$$

So $\sigma$ fulfills the definition (4), since $L(0)=[-1,1]$. To ensure (27) we recall that by the assumptions $\int_{0}^{x} f(s) d s \in[0,2]$ for $x \in[0,1]$. The uniqueness given by Theorem 1 ends our considerations. We are done.

The proof of the second part of Theorem 3 is almost the same as for the third part of Theorem 2. If $\min u<0$, then repeating the analysis from the previous proof we obtain that there exists an interval $[a, b]$ with $a<b$ such that $u$ is strictly decreasing on $[0, a]$ and $u$ is strictly increasing on $[b, 1]$. Based on (21)-(23) we find that $\int_{a}^{b} f(s) d s=2$. Next, we obtain the formula on the solution being a modification of (25) due to different boundary conditions, here $u(1)=0$. The last step is to construct a solution under condition $\int_{0}^{1} f(s) d s>2$. And here we repeat the third point from the proof of Theorem 2.

Acknowledgments. The author thanks Piotr Rybka for fruitful discussion, he is also grateful to the anonymous referees for suggesting several improvements of the paper. The author has been supported by MN grant No. N N201 268935 and by Foundation for Polish Science in fr. EU European Regional Development Funds (OPIE 2007-2013).

\section{REFERENCES}

1. Agarwal, R. P., Lü, H. \& O’Regan, D., Existence theorems for the one-dimensional singular $p$ Laplacian equation with sign changing nonlinearities. Appl. Math. Comput. 143 (2003), 15-38. Zbl1031. 34023 MR1979484

2. Ambrosetti, A. \& MAlchiodi, A., Nonlinear analysis and semilinear elliptic problems. Cambridge Studies in Advanced Mathematics, 104. Cambridge (2007). Zbl1125. 47052 MR2292344

3. Andreu, F., Ballester, C., Caselles, V., \& MaZ'on, J. M., The Dirichlet problem for the total variation flow. J. Funct. Anal. 180 (2001), 347-403. Zb10973. 35109 MR1814993 
4. Attouch, H. \& Damlamian, A., Application des mèthodes de convexité et monotonie à l'étude de certaines équations quasilineaires. Proc. Roy. Soc. Edinburgh Sect. A 79 (1977/78) 107-129. Zbl0374. 35022 MR0477473

5. Bellettini, G., Caselles, V. \& Novaga, M., The total variation flow in $R^{N}$, J. Diff. Eqns 184 (2002), 475-525. Zbl1036. 35099 MR1929886

6. Bereanu, C. \& MaWhin, J., Existence and multiplicity results for some nonlinear problems with singular phi-Laplacian. J. Differential Equations 243 (2007), 536-557. Zbl1148. 34013 MR2371799

7. Francfort, G., Murat, F. \& TARTAR, L., Monotone operators in divergence form with $x$-dependent multivalued graphs. Bollettino dell Unione Matematica Italiana, Sezione B: Articoli di Ricerca Matematica 87 (2004), 23-59. Zbl1115. 35047 MR2044260

8. Giga, M. H., Giga, Y. \& Kobayashi, R., Very singular diffusion equation. Adv. Studies Pure Math. 31 (2001), 93-125. Zbl1002.35074 MR1865089

9. Giga, Y. \& Kobayashi, R., Equations with singular diffusivity. J. Stat. Phys. 95 (1999), 1187-1220. Zb10952.74014 MR1712447

10. Giga, Y. \& RYBKA, P., Stability of facets of self-similar motion of a crystal. Adv. Differential Equations 10 (2005), 601-634. Zbl1109.35122 MR2133647

11. Giga, Y. \& RYBKA, P., Stability of facets of crystals growing from vapor. Discrete Contin. Dyn. Syst. 14 (2006), 689-706. Zbl1095.35078 MR2177092

12. Giga, Y. \& RYbKa, P., Facet bending in the driven crystalline curvature flow in the plane. J. Geom. Anal. 18 (2008), 109-147. Zbl1146.53037 MR2365670

13. Gilbarg, D. \& Trudinger, N. S., Elliptic partial differential equations of second order. SpringerVerlag, Berlin (2001). Zbl1042.35002 MR1814364

14. Guidotti, P., A new nonlocal nonlinear diffusion of image processing. J. Differential Equations 246 (2009), 4731-4742. Zb11170.35450 MR2523301

15. Gwiazda, P. \& Zatorska-Goldstein, A., On elliptic and parabolic systems with $x$-dependent multivalued graphs. Math. Methods Appl. Sci. 30 (2007), 213-236. Zbl1126. 35113 MR2285122

16. Mucha, P. B. \& RYвKA, P., A new look at equilibria in Stefan type problems in the plane. SIAM J. Math. Anal. 39 (2007), 1120-1134. Zb11145.53033 MR2368896

17. Mucha, P. B. \& RybKa, P., A caricature of a singular curvature flow in the plane. Nonlinearity 21 (2008), 2281-2316. Zbl1160.35436 MR2439480

18. SAntos, L., Strong solutions to elliptic and parabolic problems with quasilinear monotone discontinuities. Nonlinear Anal. 17 (1991), 811-824. Zb10781. 35019 MR1131491

19. Spohn, H., Surface dynamics below the roughening temperature, J. Phys. I. France 3 (1997), 69-81. 